\title{
Comparison of Automated Nucleic Acid Extraction Methods for the Detection of Cytomegalovirus DNA in Fluids and Tissues
}

Testing for cytomegalovirus (CMV) DNA is increasingly being used for specimen types other than plasma or whole blood. However, few studies have investigated the performance of different nucleic acid extraction protocols in such specimens. In this study, CMV extraction using the Cell-free 1000 and Pathogen Complex 400 protocols on the QIAsymphony Sample Processing (SP) system were compared using bronchoalveolar lavage fluid (BAL), tissue samples, and urine. The QIAsymphonyAssay Set-up (AS) system was used to assemble reactions using artus CMV PCR reagents and amplification was carried out on the RotorGene Q. Samples from 93 patients previously tested for CMV DNA and negative samples spiked with CMV AD-169 were used to evaluate assay performance. The Pathogen Complex 400 protocol yielded the following results: BAL, sensitivity $100 \%$ (33/33), specificity $87 \%$ (20/23); tissue, sensitivity $100 \%$ (25/25), specificity $100 \%$ (20/20); urine, sensitivity $100 \%$ (21/21), specificity $100 \%$ (20/20). Cell-free 1000 extraction gave comparable results for BAL and tissue, however, for urine, the sensitivity was $86 \%$ (18/21) and specimen quantitation was inaccurate. Comparative studies of different extraction protocols and DNA detection methods in body fluids and tissues are needed, as assays optimized for blood or plasma will not necessarily perform well on other specimen types. 


\section{Authors}

2 Jesse J. Waggoner

3 Department of Medicine, Division of Infectious Diseases and Geographic Medicine, Stanford

4 University School of Medicine, Stanford, CA, USA

5 Corresponding Author:

6 Benjamin A. Pinsky

7 Department of Pathology and Department of Medicine, Division of Infectious Diseases and

8 Geographic Medicine, Stanford University School of Medicine, Stanford, CA, USA.

93375 Hillview Ave, Room 2913

10 Palo Alto, CA 94304

11 Phone: 650-721-1896

12 Fax: 650-723-6918

13 E-mail: bpinsky@stanford.edu 


\section{Introduction}

15 Cytomegalovirus (CMV) is a common human viral pathogen with anywhere from $45-100 \%$ of

16 adults demonstrating serologic evidence of CMV exposure, depending on the population (Cannon

17 et al. 2010). CMV infection remains an important cause of morbidity and mortality following

18 solid organ and hematopoietic stem cell transplantation (Kotton et al. 2010; Ljungman et al.

19 2008). CMV disease has also been shown to predict treatment failure in ulcerative colitis (UC),

20 and it can cause sensorineural hearing loss and developmental abnormalities in congenitally

21 infected neonates, even when asymptomatic at birth (Lanari et al. 2006; Roblin et al. 2011).

22 Quantitative PCR testing for CMV from patient plasma or whole blood has become the standard

23 of care for detection and monitoring in transplant recipients (Caliendo et al. 2007; Kotton et al.

24 2010). The definitive diagnosis of CMV end-organ disease in immunocompromised patients

25 often requires demonstration of characteristic viral inclusions or detection of viral proteins by

26 immunohistochemical staining in tissue samples. However, PCR testing for CMV DNA in body

27 fluids and tissues is increasingly being used to aid in the diagnostic work-up for entities such as

28 CMV pneumonitis in lung transplant recipients or colonic involvement in UC (Ljungman et al.

29 2002; Riise et al. 2000; Roblin et al. 2011). In the case of congenital CMV infection, diagnosis is

30 usually made by detection of the virus in urine or saliva, although prenatal testing using amniotic

31 fluid samples in quantitative PCR or RT-PCR assays has been described (Goegebuer et al. 2009;

32 Lanari et al. 2006; Revello et al. 2003).

33 A number of comparative analyses of methods for CMV DNA detection and quantitation in

34 plasma have been published, but similar evaluations of the detection of CMV DNA in non-

35 plasma specimens are limited (Bravo et al. 2011; Caliendo et al. 2007; Fahle \& Fischer 2000;

36 Forman et al. 2011). Furthermore, it is unclear if the same nucleic acid extraction protocol used 
37 to isolate CMV DNA from plasma will be the optimum for non-plasma samples (Fahle \& Fischer

38 2000; Tang et al. 2005; Verheyen et al. 2012). In this study, we describe the performance

39 characteristics of the artus CMV PCR for the qualitative detection of CMV DNA in patient

40 bronchoalveolar lavage (BAL) fluid, tissue samples, and urine using two automated extraction

41 protocols, the Cell-free 1000 and Pathogen Complex 400, on the QIAsymphony Sample

42 Processing (SP) / Assay Set-up (AS) system and Rotor-Gene Q (RGQ). The Cell-free 1000

43 protocol is a plasma extraction protocol, whereas the Pathogen Complex 400 was designed for

44 use with serum, plasma, CSF, respiratory specimens (including BAL fluid), and urine. These

45 results are compared with those obtained using a previous laboratory standard, the COBAS

46 Amplicor CMV Monitor (CACM) assay following extraction with the MagNA Pure LC DNA

47 isolation kit. 
Materials and Methods

\section{Specimens}

50 The Stanford University Institutional Review Board waived approval for this work as it was

51 performed in the clinical virology laboratory for the purposes of quality assurance and test

52 validation.

53 Ninety-three archived patient specimens, previously tested using the CACM assay (Roche,

54 Indianapolis, IN), were evaluated. Samples were stored as aliquots at $-80^{\circ} \mathrm{C}$ following initial

55 testing. These specimens included 36 BAL (13 detected, 23 not detected), 25 tissues [5 detected

56 (3 small bowel, 2 colon), 20 not detected (5 skin, 4 small bowel, 4 colon, 3 liver, 2 lung, 1 lymph

57 node, 1 anterior mediastinal mass], and 21 urine samples (1 detected, 20 not detected). BAL

58 specimens were collected in sterile containers, and if mucoid, the samples were vortexed with

59 sterile glass beads to reduce specimen viscosity. Tissue specimens were collected in M4RT

60 (Remel, Lenexa, KS) viral transport media (VTM) or sent in a sterile container, and upon receipt,

61 transferred to Bartels Tissue Culture Refeeding Media (Trinity Biotech USA Inc., Jamestown,

62 NY) supplemented with $2 \%$ fetal bovine serum, $15 \mu \mathrm{g} / \mathrm{mL}$ gentamicin, $100 \mu \mathrm{g} / \mathrm{mL}$ vancomycin,

63 and $3.5 \mu \mathrm{g} / \mathrm{mL}$ amphotericin B. Tissues were minced using a sterile scalpel and then ground with

64 a tissue grinder.

65 Quantitated cell culture lysate, obtained from fibroblast cells infected with the AD-169 strain of

66 CMV (ATCC VR-538), was used to spike negative samples for extraction using the different

67 protocols. Cell culture lysate was quantitated using the artus CMV PCR. For BAL and tissue

68 specimens, 10-fold dilutions were prepared wherein samples were spiked to a final concentration

69 of 6.6 to $2.6 \log _{10}$ copies/mL. Four samples of each specimen type were tested at each

70 concentration. The concentration for tissue samples was calculated using the volume of media in 
71 each sample after the sample was ground. For the urine samples, four samples were tested at 10-

72 fold dilutions extending from $6.0 \log _{10}$ to $2.0 \log _{10}$ copies $/ \mathrm{mL}$. Patient specimens that had

73 previously tested positive are referred to as clinical samples to differentiate them from spiked

74 samples.

\section{Nucleic Acid Extraction and Assay Set-up}

76 For the reference method, $100 \mu \mathrm{L}$ of each specimen was extracted on the MagNA Pure LC

77 (Roche, Indianapolis, IN) using the MagNA Pure LC DNA isolation kit with the DNA I Blood

78 Cell High Performance protocol. The CACM assay was set-up manually using 50 $\mu$ L out of the $79100 \mu \mathrm{L}$ elution.

80 For the test methods, $400 \mu \mathrm{L}$ of specimen was extracted on the QIAsymphony SP (Qiagen,

81 Germantown, MD) using the Qiagen Virus/Bacteria Midi kit and Pathogen Complex 400

82 protocol. Specimens were also extracted using the same kit and the Cell-free 1000 protocol,

83 which extracts from $1000 \mu \mathrm{L}$ of sample. For eight negative tissue specimens, there was

84 insufficient volume for extraction using the Cell-free 1000 protocol. The artus CMV PCR assay

85 (Qiagen, Germantown, MD) was set-up with a custom protocol on the QIAsymphony AS that

86 utilizes $10 \mu \mathrm{L}$ of the $95 \mu \mathrm{L}$ elution, $12.5 \mu \mathrm{L}$ CMV RG Master, and $2.5 \mu \mathrm{L}$ of CMV Mg-Sol, for a

87 final reaction volume of $25 \mu \mathrm{L}$.

\section{CMV Amplification and Detection}

89 The CACM reactions utilized non-saturating, end-point PCR targeting the CMV polymerase

90 UL54 gene followed by detection via an automated enzyme-linked oligosorbent assay (ELOSA).

91 The artus CMV RG PCR reactions used a real-time, hydrolysis-probe-based PCR targeting the

92 CMV Major Immediate Early gene and were run on the RGQ. The reactions underwent 10 min 
93 at $95^{\circ} \mathrm{C}$, then 10 cycles of touchdown PCR with the annealing step starting at $65^{\circ} \mathrm{C}$ for $30 \mathrm{sec}$ and

94 decreasing by $1^{\circ} \mathrm{C}$ each cycle. Denaturation was at $95^{\circ} \mathrm{C}$ for $15 \mathrm{sec}$ and extension at $72^{\circ} \mathrm{C}$ for 20

95 sec. Touchdown PCR was followed by 35 cycles of $95^{\circ} \mathrm{C}$ for $15 \mathrm{sec}, 56^{\circ} \mathrm{C}$ for $30 \mathrm{sec}$, and $72^{\circ} \mathrm{C}$

96 for $20 \mathrm{sec}$. Data was collected on the green and yellow channels. A four-point standard curve

97 was included in each run. Amplification of an internal control added prior to extraction ensured

98 adequate extraction efficiency and the absence of inhibitors. The analytical performance of the

99 artus CMV PCR has been described previously (Waggoner et al. 2012).

100

101 Statistics

102 Basic statistical analysis was performed using Excel software (Microsoft, Redmond, WA). Paired

103 t-tests were performed using GraphPad software (GraphPad, La Jolla, CA). Shapiro-Wilk tests

104 were performed using SPSS software (IBM, Armonk, NY) to confirm the normality of the

105 distribution of the differences in the quantified CMV viral load. 


\section{Results and Discussion}

\section{Bronchoalveolar Lavage Fluid}

108 Thirty-six BAL specimens originally extracted with the MagNA Pure LC and tested on the

109 CACM were subsequently analyzed with the artus CMV RG PCR reagents on the RGQ after

110 automated extraction and assay set-up using the QIAsymphony SP/AS. An additional 20

111 negative BAL specimens were spiked with AD-169 and tested using the QIAsymphony

$112 \mathrm{SP} / \mathrm{AS} / \mathrm{RGQ}$. The artus reagents demonstrated 100\% (33/33) sensitivity when samples were

113 extracted with either the Cell-free 1000 or Pathogen Complex 400 protocols. This included 13/13

114 clinical samples and 20/20 spiked samples. Though this protocol was developed for qualitative

115 testing on non-plasma specimen types, quantified results were generated and expressed as

116 copies/mL. The artus assay was linear over the range of concentrations tested following the

117 extraction of spiked specimens with either protocol (Figure 1A and 1B), and no significant

118 difference in the quantitative results was observed following extraction with either protocol

119 (mean difference $0.05,95 \%$ CI -0.03 to $0.13, \mathrm{p}=0.19$ ).

120 The specificity for the artus reagents with the Cell-free 1000 and Pathogen Complex 400

121 protocols were $83 \%(19 / 23)$ and $87 \%(20 / 23)$, respectively. One sample was detected only using

122 the Cell-free 1000 protocol at a very low level ( 23 copies $/ \mathrm{mL})$. This specimen was negative for

123 CMV by viral culture, though the patient had been previously exposed to CMV (CMV IgG

124 positive). Additionally, there were three discrepant samples obtained from separate patients that

125 were detected by both the Pathogen Complex 400 and Cell-free 1000 protocols, but not CACM.

126 One of these patients received a CMV positive hematopoietic stem cell transplant and had a

127 history of CMV hepatitis and retinitis. CMV was quantitated in this BAL specimen at 1062

128 copies/mL using Pathogen Complex 400 and 472 copies/mL using Cell-free 1000. There was

129 limited clinical and laboratory data available for the remaining two patients. Only one had CMV 
130

131 concurrent BAL viral cultures were negative for CMV. Quantitation of CMV DNA in these

132 specimens revealed 112 and 29 copies/mL using Pathogen Complex 400 and 203 and 36

133 copies/mL using Cell-free 1000.

\section{Tissue}

135 Twenty-five previously tested tissue specimens and 20 negative tissue specimens spiked with 136 AD-169 were analyzed on the QIAsymphony SP/AS/RGQ system. The artus reagents 137 demonstrated $100 \%$ sensitivity for tissue specimens extracted using either the Cell-free 1000 or 138 Pathogen Complex 400 protocols (25/25, including 5/5 clinical samples and 20/20 spiked 139 samples). The specificity was $100 \%$ following extraction with either protocol, though only a 140 subset of samples could be tested by both protocols (Cell-free 1000, 12/12; Pathogen Complex

$141400,20 / 20)$. Quantitation of spiked tissue specimens using artus reagents showed good

142 agreement with the expected results following extraction with both protocols, and the assay was

143 linear over the range of concentrations tested (Figure 1C and 1D). No difference in the

144 quantitative results obtained using either extraction protocol was seen for the tissue samples

145 (mean difference $0.003,95 \% \mathrm{CI}-0.09$ to $0.1, \mathrm{p}=0.94$ ).

\section{Urine}

147 Twenty-one previously tested urine specimens and 20 negative urine specimens spiked with AD-

148169 were analyzed on the QIAsymphony SP/AS/RGQ system. Pathogen Complex 400 extraction 149 demonstrated $100 \%$ sensitivity (21/21, including one clinical sample and 20/20 spiked samples) 150 and 100\% specificity (20/20), whereas Cell-free 1000 extraction showed $86 \%$ sensitivity (18/21, 151 including one clinical sample and 17/20 spiked samples) and 100\% specificity (20/20). The three 152 spiked urine samples that tested negative following extraction with the Cell-free 1000 protocol 
153 had concentrations of $2.0 \log _{10}$ (two samples) and 3.0 $\log _{10}$ copies $/ \mathrm{mL}$ (one sample). Quantitation 154 of the spiked samples extracted with the Cell-free 1000 protocol revealed poor agreement with 155 the expected values (Figure 1E). In contrast, quantitation following Pathogen Complex 400 156 extraction was linear (Figure 1F). The concentration of CMV in the urine samples was, on 157 average, $0.55 \log _{10}$ copies/mL higher using the Pathogen Complex 400 extraction protocol 158 compared to the Cell-free 1000 (95\% CI 0.14 to $0.95, \mathrm{p}=0.01)$. $\mathrm{C}_{\mathrm{T}}$ values for the internal control 159 occurred later following extraction using the Cell-free 1000 protocol (mean, 27.76; standard 160 deviation, 2.27) compared to the Pathogen Complex 400 (mean, 25.08; standard deviation, 0.19; $161 \mathrm{p}<0.0001)$. 
162 CMV detection in body fluid and tissue samples is important for the diagnosis of CMV disease,

163 yet limited comparative data exist in the literature for viral nucleic acid extraction and PCR

164 protocols in such samples (Chemaly et al. 2004; Fahle \& Fischer 2000; Kotton et al. 2010). In

165 this study, we used BAL fluid, tissue samples, and urine to evaluate two DNA extraction

166 protocols utilizing the QIAsymphony SP/AS followed by real-time PCR with artus CMV PCR

167 reagents on the RGQ. Both protocols showed good agreement with CACM, the reference

168 method, for BAL and tissue specimens.

169 Quantitative testing has been reported in the literature for all of these specimen types (Chemaly et

170 al. 2004; Ganzenmueller et al. 2009; Kearns et al. 2002; Riise et al. 2000; Westall et al. 2004).

171 However, clinically meaningful thresholds for the quantitative detection CMV DNA in such

172 specimens have not yet been defined. Even with the use of a common calibrator such as the $1^{\text {st }}$

173 World Health Organization International Standard for Human Cytomegalovirus for Nucleic Acid

174 Amplification Techniques, significant concerns remain regarding sample collection and

175 processing, which cannot be standardized in a fashion similar to plasma or whole blood

176 collection. Therefore, the testing protocols described in this study were validated in our

177 laboratory for the qualitative detection of CMV from non-plasma tissue specimens. Using spiked

178 clinical specimens, quantitative CMV PCR using artus CMV reagents allowed for comparisons

179 of the efficiency of DNA extraction with the Cell-free 1000 and Pathogen Complex 400

180 protocols. The ranges of concentrations were selected to reflect those previously reported in the

181 literature, though reports are scant, particularly for tissue samples (Chemaly et al. 2004; de Vries

182 et al. 2012; Ganzenmueller et al. 2009; Kearns et al. 2002; Riise et al. 2000; Roblin et al. 2011;

183 Westall et al. 2004). Following extraction with either protocol, artus CMV PCR was linear over

184 the range of concentrations tested for BAL fluid and tissue samples, and the results showed good

185 agreement with expected values. 
186 In spiked urine samples, the Pathogen Complex 400 protocol proved more sensitive than the Cell-

187 free 1000, and the results of the artus CMV PCR following extraction with the Pathogen

188 Complex 400 were also more accurate when compared to the expected quantitative values. The

189 detection of CMV in urine samples is primarily used for the diagnosis of congenital infection

190 among newborns (Lanari et al. 2006). Despite the high viral loads often present in congenital

191 CMV, previous reports using traditional viral culture methods have missed the diagnosis in

192 symptomatic infants (Nelson et al. 1995). The severity of possible sequelae and the lack of a

193 range in CMV viral load that defines an infant's risk support the use of the most sensitive test to

194 detect CMV in newborns. In addition, an extraction protocol such as Pathogen Complex 400 that

195 allows for accurate CMV quantitation in urine may be useful in defining quantitative thresholds

196 for risk stratification.

197 Based on our results, the Cell-free 1000 extraction protocol is not appropriate for urine samples

198 as it resulted in false negatives on $3 / 20$ spiked samples. These may have resulted from the failure

199 of this protocol to remove PCR inhibitors from urine samples. This conclusion is supported by

200 the finding that internal control $\mathrm{C}_{\mathrm{T}}$ values in the artus assay were significantly higher and showed

201 greater variability following extraction with the Cell-free 1000 protocol compared to the

202 Pathogen Complex 400. These two protocols differ predominantly by the inclusion of a wash

203 step with buffer ATL (containing SDS) in the Pathogen Complex 400 protocol, and this may have

204 accounted for the improved results in urine samples. The Cell-free 1000 protocol could still be

205 used as the sole method for CMV DNA extraction in laboratories that do not test for CMV DNA

206 from urine. 
207 Three BAL samples were positive using the artus CMV PCR with either extraction protocol but 208 negative by the CACM, and a single sample was positive only after extraction using the Cell-free 2091000 protocol. On further evaluation, these samples appeared to have low positive results below 210 the limit reported by the CACM. CMV PCR and hybrid capture assays have been evaluated in 211 BAL samples from lung transplant recipients, though the clinical significance of low-positive 212 results, such as those we detected in this study, remains unclear (Chemaly et al. 2004; Riise et al. 213 2000). The quantitative results from these studies have not consistently predicted CMV

214 pneumonitis, however, no patient with a negative test for CMV had CMV pneumonitis on biopsy

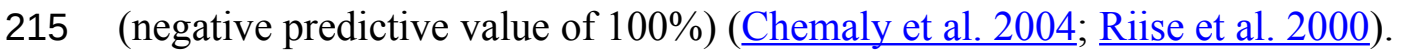




\section{Conclusions}

217 Body fluids and tissues are important specimen types for the evaluation of CMV-related diseases.

218 Reliable detection of CMV DNA in these specimen types depends not only on the sensitivity of

219 the PCR assay used but also on the extraction protocol employed in the clinical laboratory.

220 Further comparative studies of different extraction protocols and DNA detection methods in body

221 fluids and tissues are needed, as protocols optimized for plasma or whole blood cannot always be 222 applied to other specimen types. 


\section{Acknowledgments}

224 We thank the staff of the Stanford Clinical Virology Laboratory for their continued exceptional

225 work and dedication, particularly Paolo Libiran who made important contributions to this project.

226 Qiagen provided the artus CMV RG PCR reagents used in this study. 
228 Bravo D, Clari MA, Costa E, Munoz-Cobo B, Solano C, Jose Remigia M, and Navarro D. 2011. Comparative Evaluation of Three Automated Systems for DNA Extraction in Conjunction with Three Commercially Available Real-Time PCR Assays for Quantitation of Plasma

Caliendo AM, Ingersoll J, Fox-Canale AM, Pargman S, Bythwood T, Hayden MK, Bremer JW, and Lurain Cytomegalovirus DNAemia in Allogeneic Stem Cell Transplant Recipients. J Clin Microbiol 49:2899-2904.

Cannon MJ, Schmid DS, and Hyde TB. 2010. Review of cytomegalovirus seroprevalence and demographic characteristics associated with infection. Rev Med Virol 20:202-213. NS. 2007. Evaluation of real-time PCR laboratory-developed tests using analyte-specific reagents for cytomegalovirus quantification. J Clin Microbiol 45:1723-1727. Vossen AC. 2012. Real-time PCR versus viral culture on urine as a gold standard in the diagnosis of congenital cytomegalovirus infection. J Clin Virol 53:167-170.

Fahle GA, and Fischer SH. 2000. Comparison of six commercial DNA extraction kits for recovery of cytomegalovirus DNA from spiked human specimens. J Clin Microbiol 38:3860-3863. platform for nucleic acid extraction and real-time PCR assay setup. J Clin Microbiol 49:27032705.

Ganzenmueller T, Henke-Gendo C, Schlue J, Wedemeyer J, Huebner S, and Heim A. 2009. Quantification of cytomegalovirus DNA levels in intestinal biopsies as a diagnostic tool for CMV intestinal disease. J Clin Virol 46:254-258. 
253 Goegebuer T, Van Meensel B, Beuselinck K, Cossey V, Van Ranst M, Hanssens M, and Lagrou K. 2009.

254 Clinical predictive value of real-time PCR quantification of human cytomegalovirus DNA in 255 amniotic fluid samples. J Clin Microbiol 47:660-665.

256 Kearns AM, Turner AJ, Eltringham GJ, and Freeman R. 2002. Rapid detection and quantification of CMV DNA in urine using LightCycler-based real-time PCR. J Clin Virol 24:131-134.

Kotton CN, Kumar D, Caliendo AM, Asberg A, Chou S, Snydman DR, Allen U, and Humar A. 2010. International consensus guidelines on the management of cytomegalovirus in solid organ transplantation. Transplantation 89:779-795.

261 Lanari M, Lazzarotto T, Venturi V, Papa I, Gabrielli L, Guerra B, Landini MP, and Faldella G. 2006. Neonatal cytomegalovirus blood load and risk of sequelae in symptomatic and asymptomatic congenitally infected newborns. Pediatrics 117:e76-83.

Ljungman P, de la Camara R, Cordonnier C, Einsele H, Engelhard D, Reusser P, Styczynski J, and Ward K. 2008. Management of CMV, HHV-6, HHV-7 and Kaposi-sarcoma herpesvirus (HHV-8) infections in patients with hematological malignancies and after SCT. Bone Marrow Transplant 42:227-240.

Ljungman P, Griffiths P, and Paya C. 2002. Definitions of cytomegalovirus infection and disease in transplant recipients. Clin Infect Dis 34:1094-1097.

Nelson CT, Istas AS, Wilkerson MK, and Demmler GJ. 1995. PCR detection of cytomegalovirus DNA in serum as a diagnostic test for congenital cytomegalovirus infection. J Clin Microbiol 33:3317-

272 3318.

273 Revello MG, Lilleri D, Zavattoni M, Furione M, Middeldorp J, and Gerna G. 2003. Prenatal diagnosis of congenital human cytomegalovirus infection in amniotic fluid by nucleic acid sequence-based amplification assay. J Clin Microbiol 41:1772-1774.

276 Riise GC, Andersson R, Bergstrom T, Lundmark A, Nilsson FN, and Olofsson S. 2000. Quantification of cytomegalovirus DNA in BAL fluid: a longitudinal study in lung transplant recipients. Chest

278 118:1653-1660. 
Roblin X, Pillet S, Oussalah A, Berthelot P, Del Tedesco E, Phelip JM, Chambonniere ML, Garraud O, Peyrin-Biroulet L, and Pozzetto B. 2011. Cytomegalovirus load in inflamed intestinal tissue is predictive of resistance to immunosuppressive therapy in ulcerative colitis. Am J Gastroenterol 106:2001-2008.

Tang YW, Sefers SE, Li H, Kohn DJ, and Procop GW. 2005. Comparative evaluation of three commercial systems for nucleic acid extraction from urine specimens. J Clin Microbiol 43:4830-4833.

Verheyen J, Kaiser R, Bozic M, Timmen-Wego M, Maier BK, and Kessler HH. 2012. Extraction of viral nucleic acids: comparison of five automated nucleic acid extraction platforms. J Clin Virol $54: 255-259$.

Waggoner J, Ho DY, Libiran P, and Pinsky BA. 2012. Clinical significance of low cytomegalovirus DNA levels in human plasma. J Clin Micobiol 50:2378-2383.

Westall GP, Michaelides A, Williams TJ, Snell GI, and Kotsimbos TC. 2004. Human cytomegalovirus load in plasma and bronchoalveolar lavage fluid: a longitudinal study of lung transplant recipients. J Infect Dis 190:1076-1083. 


\section{Figure 1}

Assay linearity by specimen type using different extraction protocols.

Linearity of the artus CMV RG PCR assay on DNA extracted from negative clinical samples spiked with serial dilutions of AD-169 using the Cell-free 1000 and Pathogen Complex 400 protocols on the QIAsymphony SP: BAL samples (A and B), tissue samples (C and D), and urine samples ( $\mathrm{E}$ and $\mathrm{F})$. 
A.

BAL Cell-free 1000

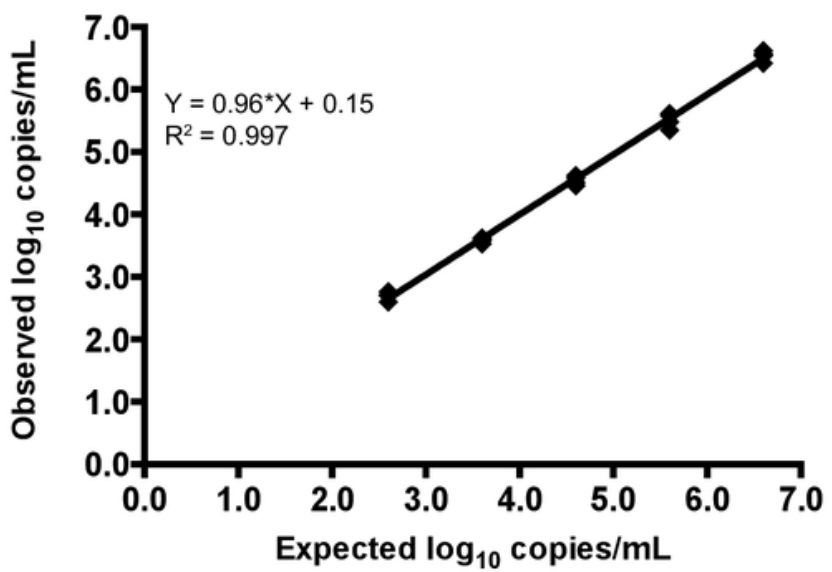

C.

Tissue Cell-free 1000

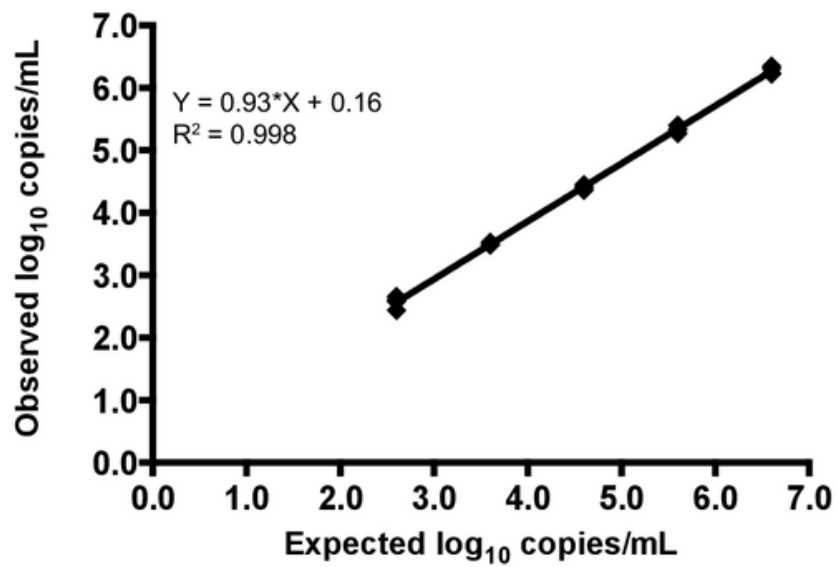

E.

Urine Cell-free 1000

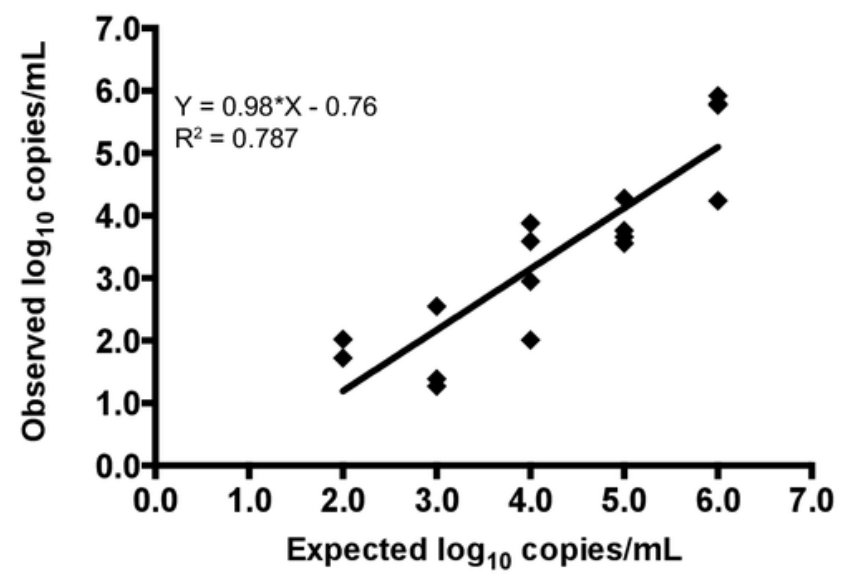

B.

BAL Pathogen Complex 400

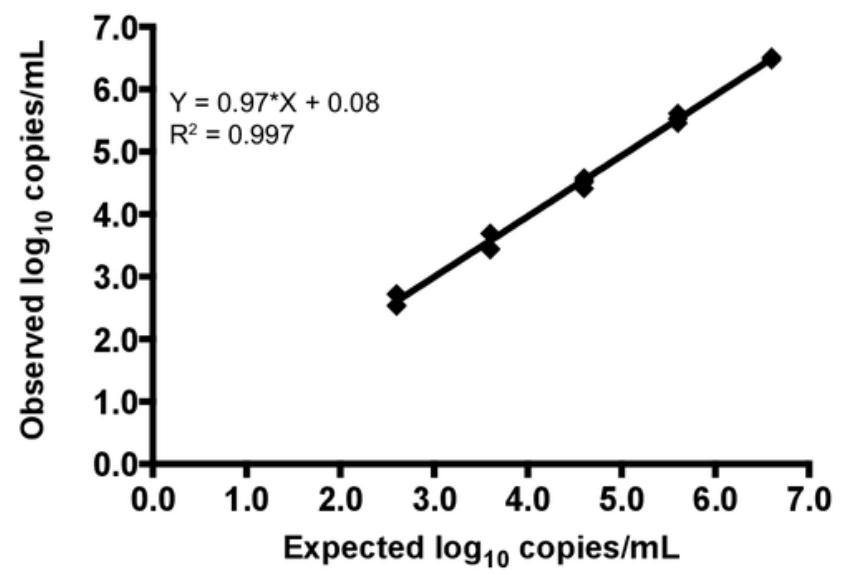

D.

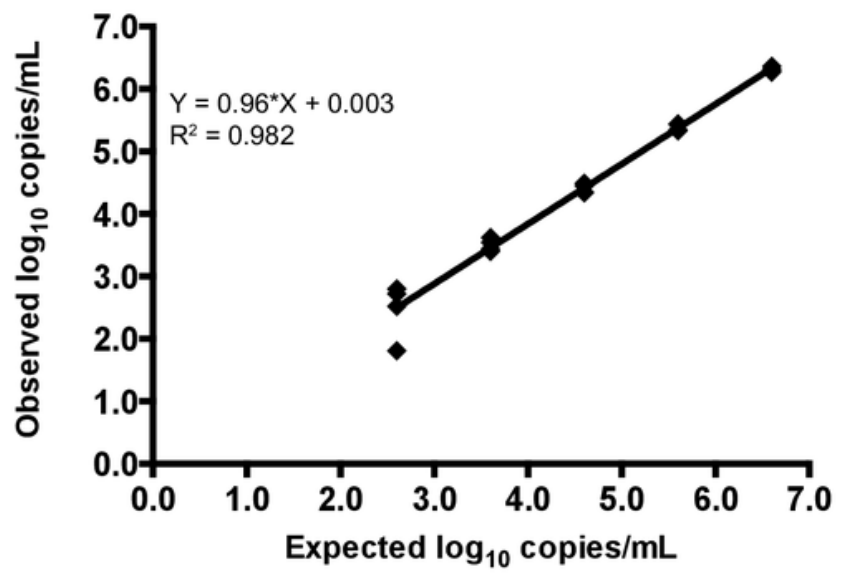

F. Urine Pathogen Complex 400

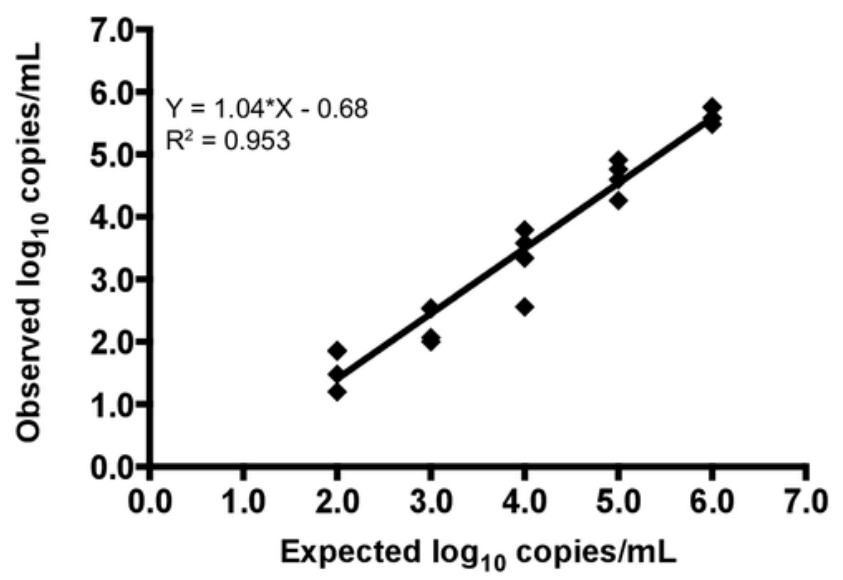

